\title{
Beamforming and Transceiver Optimization with Phase Noise for mmWave and $\mathrm{THz}$ Bands
}

\author{
Mubarak Umar Aminu, Janne Lehtomäki, and Markku Juntti \\ Centre for Wireless Communications, University of Oulu, P.O.Box 4500, FI-90014 University of Oulu, Finland \\ Email: \{firstname.lastname\}@oulu.fi
}

\begin{abstract}
This paper investigates the impact of phase noise on the transceiver signal processing originating from the local oscillators (LOs). We explore the impact of these effects with different transceiver architectures, illustrate the beam shape properties, and quantify their impact on the system performance for different modulation schemes in terms of error rates. Specifically, we model the phase noise both as Wiener and Gaussian distributed with covariance structure depending on the architecture used to share the LO signals.
\end{abstract}

\section{INTRODUCTION}

Phase noise, which is present in wireless communication systems due to imperfect oscillators, is greatly detrimental to the performance and this performance limitations is even more pronounced in systems operating at higher frequencies e.g., mmWave (30-100 GHz) and $\mathrm{THz}$ (above $300 \mathrm{GHz}$ ) bands [1], [2]. We focus on the impact of phase noise for systems operating at mmWave and $\mathrm{THz}$ regimes. To be able to fulfill the ever increasing mobile data traffic, the first standards of $5 \mathrm{G}$ explore the mmWave bands for data communications, and $\mathrm{THz}$ bands are envisaged for systems beyond $5 \mathrm{G}$.

A particularly attractive feature at mmWave and $\mathrm{THz}$ frequencies is the huge available contiguous bandwidth, which enables very short symbol intervals, and, thus, extreme data rates. Furthermore, the tiny operating wavelength allows for tightly packed large-scale antenna arrays at the transceiver, making it feasible to realize narrow pencil-like beams enabling the signal to propagate a reasonable distance. These beams can be formed using different transceiver architectures. So far, the most promising approaches are either fully analog beamforming, hybrid beamforming with a small number of RF chains, or hybrid/fully digital beamforming with low-resolution digitalto-analog converters due to their lower complexity [3]-[5]. The first one applies only low-complexity analog phase shifters to adjust the beam direction in addition to electromagnetically directive antenna elements, while the other ones use also digital processing to enhance the beamforming capability.

Phase noise (PN) caused by local oscillators (LOs) in the frequency up-conversion and down-conversion stages at the transmitter and receiver, respectively, introduces some random changes in the beam shape properties of a directed beam which consequently degrades the effective communication channel thereby the capacity of the system. Moreover, the PN causes multiplicative disturbance on the signal, resulting in rotation of the symbols in the constellation and consequently leading to symbol errors. This phase variation may be compensated for to some extent, but it becomes more and more challenging when the beams become narrower and the symbol interval decreases.

In many previous works [6]-[10], free-running oscillator and phased-locked loop based oscillators are the most common assumptions. When the system is phased-locked, the resulting PN is low and modelled as a zero mean stationary random process. In the free-running case, the system is said to be frequency-locked and the resulting PN is modeled as a Wiener process with Gaussian distributed increments between each sampling instant [8]. In multi-antenna systems, the PN can be correlated or even fully uncorrelated between different radio frequency $(\mathrm{RF})$ chains depending on the transceiver architecture and implementation [11], [12]. When all the RF chains at the transceiver share a common $\mathrm{LO}$, the $\mathrm{PN}$ is correlated among the RF chains thereby all the chains experience the same rotation. On the other hand, the $\mathrm{PN}$ is uncorrelated when each RF chain is equipped with independent LO.

In this paper, we study the impact of phase noise in a multi-antenna hybrid digital-analog beamforming transceiver architecture. We investigate the effects of phase noise to the beam properties with different LO architectures at the transceiver. We model the phase noise using three correlation models to approximate the LO architectures and evaluate their impact on the communications performance. We study the cases of common LO (CLO) shared among all the RF chains, independent LO (ILO) for each RF chain and a block-based LO (m-block LO) architecture. We study the impact on the main lobe gain, the side lobe gain, and the beam pointing error due to the PN. By means of simulations, we further study how the PN from different LO architectures affect the performance of two modulation schemes: (i) coherent quadrature phase shift keying (QPSK) and (ii) differential QPSK (DQPSK). We specifically analyze the effects in terms of signal constellation and bit error rate (BER).

\section{System And Phase Noise Model}

\section{A. Signal Model}

Consider a hybrid digital-analog two stage beamforming MIMO system as depicted in Fig. 1. The transmitter is equipped with $N_{\mathrm{Tx}}$ transmit antennas and $L_{\mathrm{Tx}} \leq N_{\mathrm{Tx}} / M_{t} \mathrm{RF}$ chains, where $M_{t}$ is the number of antennas per each subarray. Similarly, the receiver is equipped with $N_{\mathrm{Rx}}$ transmit antennas and $L_{\mathrm{Rx}} \leq N_{\mathrm{Rx}} / M_{r} \mathrm{RF}$ chains, where $M_{r}$ is the number of antennas per each subarray. The transmitted symbols are 


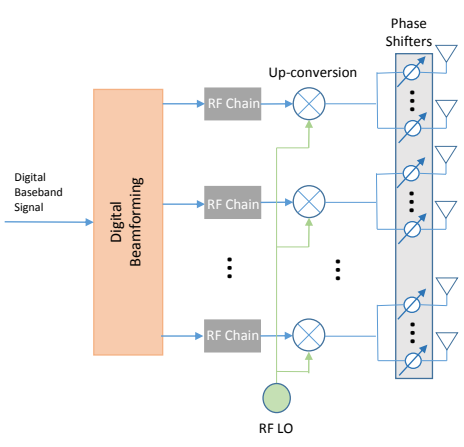

(a) Common LO

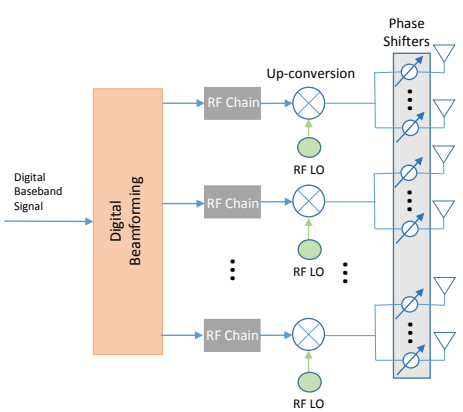

(b) Independent LO

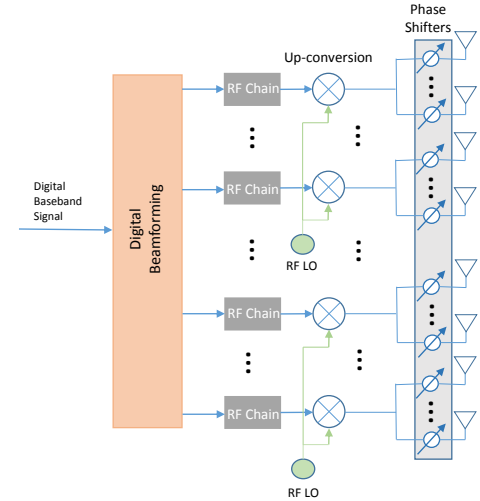

(c) Block-based LO

Fig. 1. Different transceiver LO architectures

first precoded in digital domain, up-converted, and then fed into the subarrays that further use phase shifters for analog beamforming. Let us denote $\mathbf{F}_{D} \in \mathbb{C}^{L_{\mathrm{Tx}} \times 1}$ as digital precoder, and $\mathbf{F}_{A} \in \mathbb{C}^{N_{\mathrm{Tx}} \times L_{\mathrm{Tx}}}$ as block-diagonal analog beamforming matrix at the transmitter. Then, the transmitted signal at the RF front end can be written as

$$
\begin{aligned}
\mathbf{x}[m] & =\mathbf{F}_{A} \boldsymbol{\Theta}_{\mathrm{Tx}}[m] \mathbf{F}_{D} s[m] \\
& =\tilde{\mathbf{f}} s[m]
\end{aligned}
$$

where $s[m]$ is the $m$ th transmitted symbol, and $\boldsymbol{\Theta}_{\mathrm{Tx}}[m]=$ $\operatorname{diag}\left(e^{\theta_{1}^{\mathrm{Tx}}[m]}, \ldots, e^{\theta_{L_{\mathrm{Tx}}^{\mathrm{Tx}}}^{\mathrm{x}}[m]}\right) \in \mathbb{C}^{L_{\mathrm{Tx}} \times L_{\mathrm{Tx}}}$ is the diagonal matrix involving the phase noise component for each RF chain at the transmitter. The transmitted signal $\mathbf{x}[\mathrm{m}]$ propagates through the channel $\mathbf{H} \in \mathbb{C}^{N_{\mathrm{Rx}} \times N_{\mathrm{Tx}}}$ and is received at the receiver by $N_{\mathrm{Rx}}$ antennas. The signal is received in the analog domain using phase shifter weights, down-converted to the baseband, and then combined using the digital combiner. Let $\mathbf{W}_{A} \in \mathbb{C}^{N_{\mathrm{Rx}} \times L_{\mathrm{Rx}}}$ denote the block-diagonal analog beamforming matrix at the receiver, and $\mathbf{W}_{D} \in \mathbb{C}^{L_{\mathrm{Rx}} \times 1}$ denote the digital combiner. The resulting $m$ th received symbol is expressed as

$$
r[m]=\tilde{\mathbf{w}}^{\mathrm{H}} \mathbf{H} \tilde{\mathbf{f}} s[m]+\tilde{\mathbf{w}}^{\mathrm{H}} z[m],
$$

where $(.)^{\mathrm{H}}$ is the conjugate transpose, $\tilde{\mathbf{w}}=\mathbf{W}_{A} \boldsymbol{\Theta}_{\mathrm{Rx}}[m] \mathbf{W}_{D}$, and $\boldsymbol{\Theta}_{\mathrm{Rx}}[m]=\operatorname{diag}\left(e^{\theta_{1}^{\mathrm{Rx}}[m]}, \ldots, e^{\theta_{L_{\mathrm{Rx}}}^{\mathrm{Rx}}[m]}\right) \in \mathbb{C}^{L_{\mathrm{Rx}} \times L_{\mathrm{Rx}}}$ is the diagonal matrix involving the phase noise component for each RF chain at the receiver.

\section{B. Oscillator Phase Noise}

PN introduced by oscillators essentially means that not all the power is concentrated on the single desired frequency, but spreads around the center frequency. This translates to jitter in the time domain. When the LO in the system is phase-locked i.e., phased-locked loop (PLL) is employed in the system, the PN causes a small phase mismatch and is normally well modeled by a Gaussian distribution [8]. If the system is frequency-locked, i.e., the LO in the system is tuned to the carrier but it is free-running, the $\mathrm{PN}$ in this case is modeled as a Wiener process, i.e.,

$$
\theta_{l}[m]=\theta_{l}[m-1]+w_{l}[m] \quad l=1, \ldots, L,
$$

where $w_{l}[m]$ is a Gaussian random variable. In multi-antenna system, the PN samples can be correlated among the RF chains depending on how the LO signal is shared among the RF chains at the transceiver. In this paper, we consider three different LO configurations as shown in Fig. 1. For the considered configuration, the PN samples can be modeled assuming a correlation model between the RF chains. The PN samples can be generated from a multivariate Gaussian distribution $\mathcal{N}(\mathbf{0}, \Sigma)$, where zero-vector $\mathbf{0}$ is the mean and $\Sigma$ is the $L \times L$ covariance matrix which can be written as

$$
\Sigma=\left[\begin{array}{ccc}
\sigma_{1}^{2} & \cdots & \rho \\
\vdots & \ddots & \vdots \\
\rho & \cdots & \sigma_{L}^{2}
\end{array}\right]
$$

where $\rho$ is the correlation coefficient and $\sigma_{l}^{2}$ is the elementwise variance. The correlation coefficient $\rho$ takes value either 0 or 1, i.e., $\rho=0$ means the phase noise between the corresponding RF chain is uncorrelated or when $\rho=1$ the phase noise is correlated. The examples of the covariance matrices for the common LO, independent LO and the blockbased LO architecture with 4 RF chains are given as

$$
\underbrace{\left[\begin{array}{llll}
1 & 1 & 1 & 1 \\
1 & 1 & 1 & 1 \\
1 & 1 & 1 & 1 \\
1 & 1 & 1 & 1
\end{array}\right]}_{\text {Common LO }} \underbrace{\left[\begin{array}{llll}
1 & 0 & 0 & 0 \\
0 & 1 & 0 & 0 \\
0 & 0 & 1 & 0 \\
0 & 0 & 0 & 1
\end{array}\right]}_{\text {Independent LO }} \underbrace{\left[\begin{array}{llll}
1 & 1 & 0 & 0 \\
1 & 1 & 0 & 0 \\
0 & 0 & 1 & 1 \\
0 & 0 & 1 & 1
\end{array}\right]}_{\text {Block-based LO }},
$$

and the corresponding PN matrix $\Theta$ for the configurations is expressed below at the top of the next page. 


$$
\boldsymbol{\Theta}_{\mathrm{CLO}}=\underbrace{\left[\begin{array}{cccc}
e^{j \theta} & 0 & 0 & 0 \\
0 & e^{j \theta} & 0 & 0 \\
0 & 0 & e^{j \theta} & 0 \\
0 & 0 & 0 & e^{j \theta}
\end{array}\right]}_{\text {Common LO }} \quad \Theta_{\mathrm{ILO}}=\underbrace{\left[\begin{array}{cccc}
e^{j \theta_{1}} & 0 & 0 & 0 \\
0 & e^{j \theta_{2}} & 0 & 0 \\
0 & 0 & e^{j \theta_{3}} & 0 \\
0 & 0 & 0 & e^{j \theta_{4}}
\end{array}\right]}_{\text {Independent LO }} \quad \boldsymbol{\Theta}_{2-\text { blockLO }}=\underbrace{\left[\begin{array}{cccc}
e^{j \theta_{1}} & 0 & 0 & 0 \\
0 & e^{j \theta_{1}} & 0 & 0 \\
0 & 0 & e^{j \theta_{2}} & 0 \\
0 & 0 & 0 & e^{j \theta_{2}}
\end{array}\right]}_{\text {Block-based LO }}
$$

\section{NumERICAL ANALYSIS AND EXAMPLES}

In this section, we present some numerical analysis and examples of the impact of PN on beamforming and signal constellation for two modulation schemes: QPSK and DQPSK.

\section{A. Simulation setup}

We consider a setting with $N_{\mathrm{Tx}}=32$ antennas and $L_{\mathrm{Tx}}=$ $8 \mathrm{RF}$ chains at the transmitter and $N_{\mathrm{Rx}}=32$ antennas and $L_{\mathrm{Rx}}=8 \mathrm{RF}$ chains at the receiver. We assume the line of sight scenario and the channel is modeled as the commonly used uniform linear array (ULA) channel model [13]

$$
\mathbf{H}=\alpha \mathbf{a}_{\mathrm{Rx}} \mathbf{a}_{\mathrm{Tx}}^{\mathrm{H}},
$$

where $\alpha_{k} \sim \mathcal{C N}(0,1)$ is the complex channel gain; $\mathbf{a}_{\mathrm{Rx}}$ and $\mathbf{a}_{\mathrm{Tx}}$ are the antenna array responses at the transmit and receive arrays respectively expressed as

$$
\begin{aligned}
& \mathbf{a}_{\mathrm{Rx}}=\left[1, e^{j \mu^{\mathrm{Rx}}}, \ldots, e^{j\left(\mathrm{~N}_{\mathrm{Rx}}-1\right) \mu^{\mathrm{Rx}}}\right]^{T}, \\
& \mathbf{a}_{\mathrm{Tx}}=\left[1, e^{j \mu^{\mathrm{Tx}}}, \ldots, e^{j\left(\mathrm{~N}_{\mathrm{Tx}}-1\right) \mu^{\mathrm{Tx}}}\right]^{T},
\end{aligned}
$$

where $\mu^{\mathrm{Rx}}=\frac{2 \pi}{\lambda} d \sin \left(\psi^{\mathrm{Rx}}\right), \mu^{\mathrm{Tx}}=\frac{2 \pi}{\lambda} d \sin \left(\psi^{\mathrm{Tx}}\right), \lambda$ is the carrier wavelength, $d$ is the spacing between the antenna elements, $\psi^{\mathrm{Rx}}$ and $\psi^{\mathrm{Tx}}$ are the angle of arrival and angle of the departure respectively.

\section{B. Impact of PN on Beamforming}

The array factor can be used to examine mathematically the impact of the PN on the beam response, since the array factor represents the far-field radiation pattern of an array of isotropically radiating elements. The array factor for the hybrid beamformer is given by

$$
\begin{aligned}
A F(\varphi) & =\tilde{\mathbf{f}}^{\mathrm{H}} \mathbf{a}(\varphi) \\
& =\sum_{n=1}^{N} e^{j\left(2 \pi / \lambda d n \sin \Gamma-2 \pi / \lambda d n \sin \varphi+\theta_{n}\right)},
\end{aligned}
$$

where $\varphi$ is the azimuth angle of observation, $\lambda$ is the carrier wavelength, $d$ is the spacing between the antenna elements, $\Gamma$ is the angle to which the beam is directed, $n$ is the antenna index and $\theta_{n}$ is the PN sample at $n$th antenna. Notice that in the common LO case, the PN sample across the antennas is the same i.e., $\theta_{n}=\theta \forall n$ and therefore the array factor (7) can be written as

$$
A F(\varphi)=e^{j \theta} \sum_{n=1}^{N} e^{j(2 \pi / \lambda d n \sin \Gamma-2 \pi / \lambda d n \sin \varphi)} .
$$

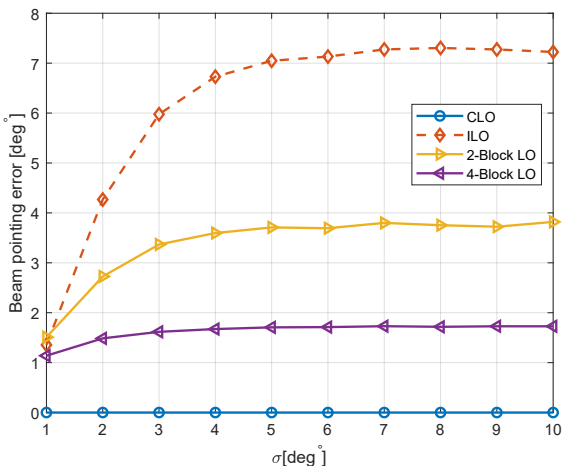

Fig. 2. Antenna main beam pointing error vs. phase noise variance.

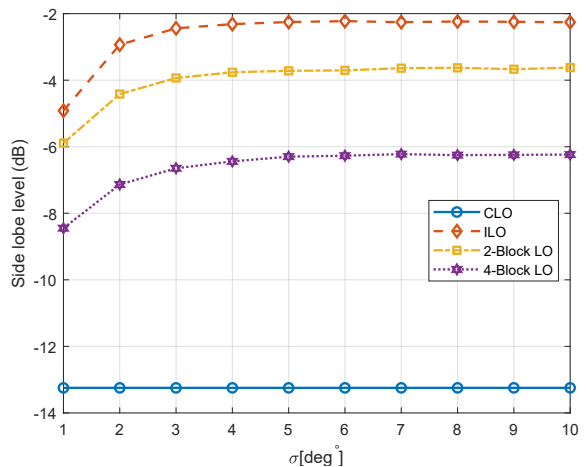

Fig. 3. Relative antenna side lobe level vs. phase noise variance.

In Fig. 4, we plotted the beam response for different values of PN variance i.e., $\sigma^{2}=\left[1^{\circ}, 3^{\circ}, 5^{\circ}, 7^{\circ}, 9^{\circ}\right]$. As can be seen in Fig. 4(a) where the PN is common among the RF chains, it causes no distortions in the beam pattern. This is because the progressive phase shift remains the same between all the antenna elements, and thus there is no error in the array factor. In Figs. 4(b) and 4(c), we can see in the case where PN is uncorrelated among the RF chains, it leads to increased side lobe levels and beam pointing errors. When the PN is Gaussian, there is no impact on the main lobe direction but PN leads to increased side lobe levels as can be seen in Fig. 4(b) where each two RF chain share a common LO. The PN, when modelled as a Wiener process, affects more severely on the beam pattern. This can be observed in Fig. 4(c). In Figs. 2 and 3 we show the beam pointing error and side lobe level respectively for the range of $\mathrm{PN}$ variance with the $\mathrm{PN}$ modeled as a Wiener process. As can be seen from the figures, the beam deviates from the desired direction with increased side lobe levels as the PN among the RF chains are uncorrelated. 


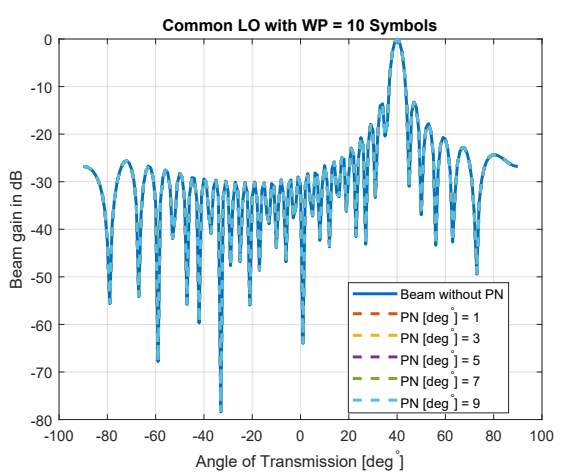

(a) $\rho=1 \mathrm{WP}(10$ symbols $)$

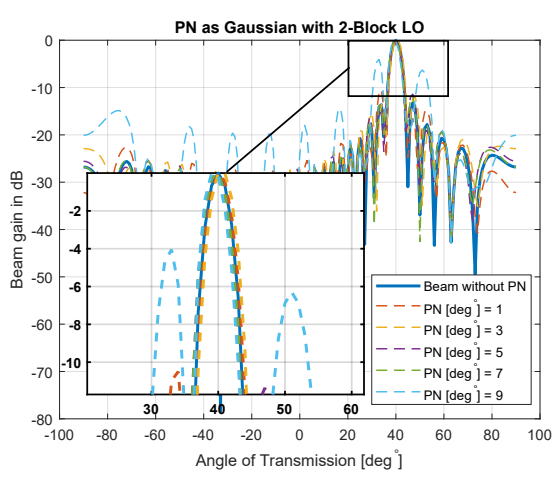

(b) PN as Gaussian

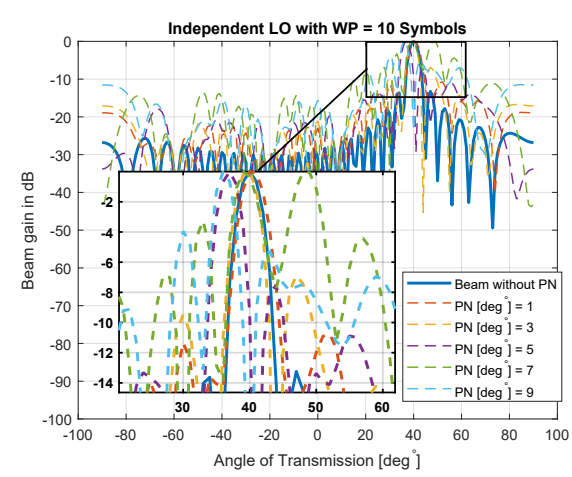

(c) PN as WP (10 symbols)

Fig. 4. Antenna beam pattern in the presence of phase noise for different numbers of symbols over which phase noise accumulates before synchronization.

In an ideal system without the impact of PN, the transmit and receive hybrid beamformers can be designed using an approach similar to that in [14]. The hybrid beamformers can be designed by solving

$$
\begin{gathered}
\left(\mathbf{F}_{A}^{\text {opt }}, \mathbf{F}_{D}^{\text {opt }}\right)=\underset{\substack{\mathbf{F}_{A} \mathbf{F}_{D} \\
\text { s.t. }}}{\operatorname{argmax}}\left\|\mathbf{f}_{A} \in \mathcal{F}_{\mathrm{RF}}-\mathbf{F}_{A} \mathbf{F}_{D}\right\|_{F}, \\
\left\|\mathbf{F}_{A} \mathbf{F}_{D}\right\|^{2}=1 .
\end{gathered}
$$

The fact that the RF precoders $\mathbf{F}_{A} \in \mathbb{C}^{N_{\mathrm{Tx}} \times L_{\mathrm{Tx}}}$ are phase shifter weigths with constant-magnitude, the following equivalent problem can be solved for the beamformers

$$
\begin{gathered}
\mathbf{F}_{D}^{\text {opt }}=\underset{\mathbf{F}_{D}}{\operatorname{argmax}}\left\|\mathbf{f}_{\text {opt }}-\mathbf{A}_{t} \mathbf{F}_{D}\right\|_{F}, \\
\text { s.t. } \quad\left\|\operatorname{diag}\left(\mathbf{F}_{D} \mathbf{F}_{D}^{\mathrm{H}}\right)\right\|_{0}=L_{\mathrm{Tx}}, \\
\quad\left\|\mathbf{A}_{t} \mathbf{F}_{D}\right\|^{2}=1,
\end{gathered}
$$

where $\mathbf{A}_{t}=\left[\mathbf{a}_{t}(0), \mathbf{a}_{t}\left(\frac{2 \pi}{Q}\right), \ldots, \mathbf{a}_{t}\left(\frac{2 \pi}{Q}(Q-1)\right)\right]$ is a matrix containing the array response vectors. Assuming perfect knowledge of the channel and the singular value decomposition (SVD) of $\mathbf{H}$ is defined as

$$
\mathbf{H}=\mathbf{U} \boldsymbol{\Lambda} \mathbf{V},
$$

where $\mathbf{U}=\left[\mathbf{u}_{1} \ldots \mathbf{u}_{N_{\mathrm{Rx}}}\right] \in \mathbb{C}^{N_{\mathrm{Rx}} \times N_{\mathrm{Rx}}}$ and $\mathbf{V}=$ $\left[\mathbf{v}_{1} \ldots \mathbf{v}_{N_{\mathrm{Tx}}}\right] \in \mathbb{C}^{N_{\mathrm{Tx}} \times N_{\mathrm{Tx}}}$ are the two unitary matrices, $\boldsymbol{\Lambda}=$ $\operatorname{diag}\left(\lambda_{1}, \lambda_{2}, \ldots, \lambda_{R}\right)$ whose diagonal elements are the singular values, and $R=\min \left(N_{\mathrm{Tx}}, N_{\mathrm{Rx}}\right)$, then optimal precoder is $\mathbf{f}_{\mathrm{opt}}=\mathbf{v}_{1}$. Problem (10) can be solved using the orthogonal matching pursuit (OMP) algorithm given in [14, Algorithm 1]. Fig. 5 shows the achievable rate with the impact of PN. It can be seen that when there is a common $\mathrm{LO}$ among the RF chains there is no impact on the beamformers thereby the capacity is not degraded. This is validated by the fact that when the PN is correlated among the RF chains, the beam pattern is not distorted as shown in Fig. 4(a). In the independent LO case, the hybrid beamformers should be designed taking into consideration of the PN. This can be incorporated into the optimization problem (10). One possible way is to solve

$$
\begin{gathered}
\mathbf{F}_{D}^{\text {opt }}=\underset{\mathbf{F}_{D}}{\operatorname{argmax}}\left\|\mathbf{f}_{\text {opt }}-\mathbf{A}_{t} \boldsymbol{\Theta}_{\mathrm{Tx}} \mathbf{F}_{D}\right\|_{F}, \\
\text { s.t. }\left\|\operatorname{diag}\left(\mathbf{F}_{D} \mathbf{F}_{D}^{\mathrm{H}}\right)\right\|_{0}=L_{\mathrm{Tx}}, \\
\quad\left\|\mathbf{A}_{t} \boldsymbol{\Theta}_{\mathrm{Tx}} \mathbf{F}_{D}\right\|^{2}=1,
\end{gathered}
$$

where $\Theta_{\mathrm{Tx}}$ is the diagonal matrix involving the PN samples. The same problem can be solved for the receive hybrid beamformers.

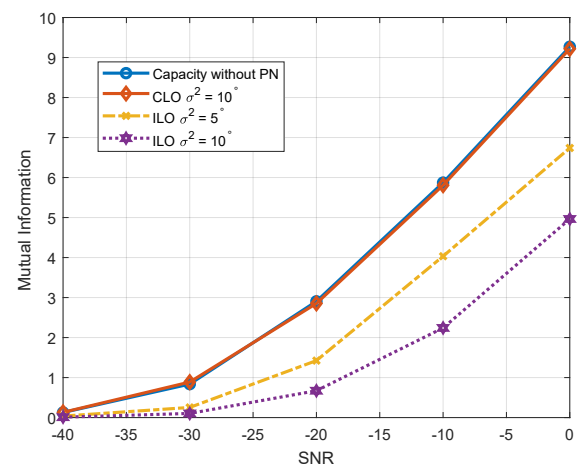

Fig. 5. Mutual information with PN modeled as Wiener process.

\section{Impact of PN on Signal Constellation}

When the PN is common among the RF chains, the effect is similar as in a one-antenna system and there is no impact on the beam pattern as shown in Fig. 4(a). This however is not the case at the baseband. The PN in the common LO case causes rotation in the received symbol. The $m$ th received signal (2) for the common LO case can be expressed as

$$
r[m]=e^{\theta_{\mathrm{Rx}}[m]+\theta_{\mathrm{Tx}}[m]} \underbrace{\mathbf{w}^{\mathrm{H}} \mathbf{H f} s[m]+\mathbf{w}^{\mathrm{H}} z[m]}_{r_{\text {ideal }}} .
$$

From (12), depending on the design of the beamfomers, the received signal is a rotated scaled version of the transmitted symbol $s[m]$ plus some additive Gaussian noise. This rotation 
can be seen in the constellation diagram in Figs. 6(b), 6(d) for QPSK and DQPSK schemes respectively. When independent LOs are used in the RF chains, the PN leads to clouds resembling impact of additive white Gaussian like noise in the constellation. The BER for different PN variance was plotted in Fig. 7. QPSK scheme degrades more when the PN is correlated among the RF chains. The DQPSK on the other hand shows to be less affected in terms of the BER when the PN are correlated among the RF chains. In the general performance, the DQPSK is more immune to the PN as compared to the QPSK scheme.

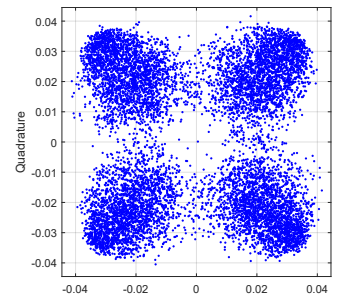

(a) QPSK with ILO

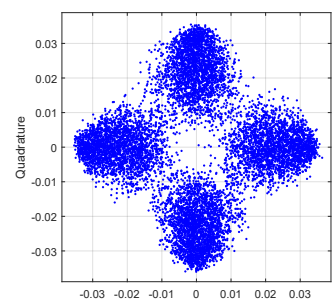

(c) DQPSK with ILO

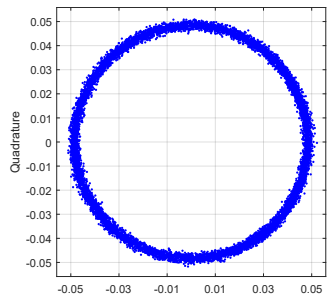

(b) QPSK with CLO

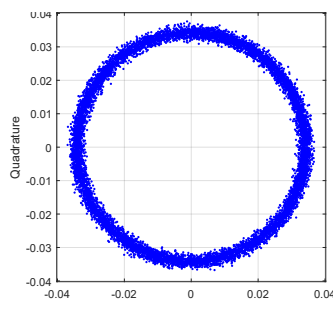

(d) DQPSK with CLO
Fig. 6. Signal constellation under $\mathrm{PN}$ with variance $2^{\circ}$

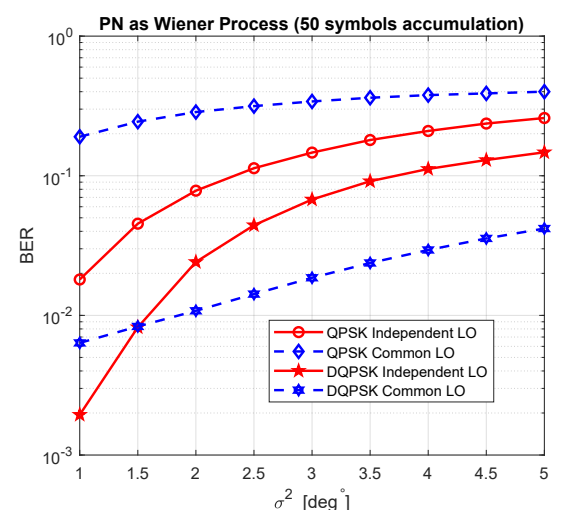

Fig. 7. BER vs. phase noise variance

\section{CONCLUSION}

We studied the impact of phase noise in a multi-antenna hybrid transceiver architecture. We investigated the effects of phase noise on the beamforming and quantified their impact on the system performance for QPSK and DQPSK. We showed how the beam pattern is affected for different $\mathrm{LO}$ architectures. We studied the cases of common LO shared among all the RF chains, the case of independent LO for each RF chain and a block-based LO (m-block LO) architecture. The results demonstrate that as the PN processes among the RF are uncorrelated, it leads to beam pointing errors and increased side lobe levels. This is important finding for multi-user systems to be considered in the future work. We further found out that the PN does not cause distortion on the beam but it causes symbol rotation in the constellation, when the $\mathrm{PN}$ is correlated among the RF chains. On the other hand, when the $\mathrm{PN}$ is uncorrelated among the RF chains, it degrades the beam pattern and causes clouds in the constellation. The results also illustrate the error rate performance of the QPSK and DQPSK, when the PN is correlated or uncorrelated. It was shown that DQPSK scheme outperforms QSPK in terms of BER with phase noise by several dBs.

\section{ACKNOWLEDGMENT}

This work has received funding from the European Commission's Horizon 2020 research and innovation programme under grant agreement No 761794 (TERRANOVA) and from Academy of Finland 6Genesis Flagship (grant 318927).

\section{REFERENCES}

[1] H. Mehrpouyan, M. R. Khanzadi, M. Matthaiou, A. M. Sayeed, R. Schober, and Y. Hua, "Improving bandwidth efficiency in E-band communication systems," IEEE Commun. Mag., vol. 52, no. 3, pp. 121128, 2014.

[2] P. Smulders et al., "Exploiting the $60 \mathrm{GHz}$ band for local wireless multimedia access: prospects and future directions," IEEE Commun. Mag., vol. 40, no. 1, pp. 140-147, 2002.

[3] A. Alkhateeb, J. Mo, N. Gonzalez-Prelcic, and R. W. Heath, "MIMO precoding and combining solutions for millimeter-wave systems," IEEE Commun. Mag., vol. 52, no. 12, pp. 122-131, 2014

[4] A. F. Molisch, V. V. Ratnam, S. Han, Z. Li, S. L. H. Nguyen, L. Li, and K. Haneda, "Hybrid beamforming for massive MIMO: A survey," IEEE Commun. Mag., vol. 55, no. 9, pp. 134-141, 2017.

[5] S. Han, I. Chih-Lin, Z. Xu, and C. Rowell, "Large-scale antenna systems with hybrid analog and digital beamforming for millimeter wave 5G," IEEE Commun. Mag., vol. 53, no. 1, pp. 186-194, 2015.

[6] D. Petrovic, W. Rave, and G. Fettweis, "Effects of phase noise on OFDM systems with and without PLL: Characterization and compensation," IEEE Trans. Commun., vol. 55, no. 8, pp. 1607-1616, 2007.

[7] S. Wu and Y. Bar-Ness, "OFDM systems in the presence of phase noise: consequences and solutions," IEEE Trans. Commun., vol. 52, no. 11, pp. 1988-1996, 2004.

[8] L. Piazzo and P. Mandarini, "Analysis of phase noise effects in OFDM modems," IEEE Trans. Commun., vol. 50, no. 10, pp. 1696-1705, 2002.

[9] D. S. Baum and H. Bolcskei, "Impact of phase noise on MIMO channel measurement accuracy," in 2004 IEEE 60th Vehicular Technology Conference, 2004. VTC2004-Fall., vol. 3. IEEE, 2004, pp. 1614-1618.

[10] A. Taparugssanagorn and J. Ylitalo, "Characteristics of short-term phase noise of MIMO channel sounding and its effect on capacity estimation," IEEE Trans. Instrum. Meas., vol. 58, no. 1, pp. 196-201, 2009.

[11] H. Thomas, V. Ranki et al., "Phase noise in beamforming," IEEE Trans. Wireless Commun., vol. 9, no. 12, pp. 3682-3689, 2010.

[12] X. Chen, H. Wang, W. Fan, Y. Zou, A. Wolfgang, T. Svensson, and J. Luo, "Phase noise effect on MIMO-OFDM systems with common and independent oscillators," Wireless Communications and Mobile Computing, vol. 2017, 2017.

[13] M. R. Akdeniz, Y. Liu, M. K. Samimi, S. Sun, S. Rangan, T. S. Rappaport, and E. Erkip, "Millimeter wave channel modeling and cellular capacity evaluation," IEEE journal on selected areas in communications, vol. 32, no. 6, pp. 1164-1179, 2014.

[14] O. El Ayach, S. Rajagopal, S. Abu-Surra, Z. Pi, and R. W. Heath, "Spatially sparse precoding in millimeter wave MIMO systems," IEEE Trans. Commun., vol. 13, no. 3, pp. 1499-1513, 2014. 